\title{
Grand challenges of structural sensing
}

\author{
lan F. C. Smith* \\ École Polytechnique Fédérale de Lausanne, Lausanne, Switzerland
}

Keywords: sensing, structural sensing, structures, construction, prediction

In spite of our huge global investment in structures and infrastructure (tens of trillions of dollars annually), structural sensing is in its infancy. Much less than $1 \%$ of all structures in the built environment are measured. While sensors that are appropriate for the context of structures in the built environment have been around for decades, and some measurement technologies have even been available for centuries, engineers in this field have had to wait for cheap, robust, and portable data acquisition and storage technology. From the 1990s, this potential increased further with the introduction of fiber-optic technologies, thereby providing the means to observe long-term behavior, such as creep, temperature effects, corrosion, and fatigue cracking in non-homogenous materials and other anomalies in structural behavior.

If all this has happened, why is structural sensing still in its infancy? This is because there are many more grand challenges left to meet. Some of these are described next.

Structural sensing has the potential to increase knowledge and improve engineering decision making during the following engineering activities:

OPEN ACCESS

Edited by:

Branko Glisic,

Princeton University, USA

Reviewed by:

Matthew Yarnold,

Tennessee Technological University,

USA

*Correspondence:

lan F. C. Smith

ian.smith@epfl.ch

Specialty section:

This article was submitted to

Structural Sensing, a section of the

journal Frontiers in Built Environment

Received: 08 September 2015

Accepted: 22 September 2015

Published: 07 October 2015

Citation:

Smith IFC (2015) Grand challenges of structural sensing.

Front. Built Environ. 1:19. doi: 10.3389/fbuil.2015.00019
- laboratory research into new materials, new material combinations, new structural elements, new connections, and new structural systems;

- construction phases where there are high risks and doubts about real structural behavior;

- in-service measurement at early stages in the life cycle when no damage is present;

- in-service measurement to assess the effects of damage and occasionally, to detect new damage;

- during dismantlement and demolition where there are high risk stages;

- construction and use of active structures for use in challenging environments.

The next paragraphs contain more detailed descriptions of these activities within the context of the built environment. For each activity, important challenges that need to be met are identified. The article finishes with a discussion of grand challenges that are not specifically related to individual activities.

Scientific developments are often preceded by developments of new ways to measure phenomena. In the laboratory, structural sensing has increased our knowledge of behavior for all limit states of structural elements, connections, structures, and structural systems in the built environment. Design specifications, codes and standards are continually improved as a result of this research. Since this work is carried out in controlled environments in this context, sensors do not need to be either particularly rugged or have long life spans. Accuracy and precision are important and usually, there is a current measurement technology that is available to meet most research needs. Perhaps, the biggest challenge for this activity is correctly matching the technology, along with its costs, to the needs of the investigation.

This activity is structural sensing's lone success story in terms of the percentage use within the activities listed earlier. It is almost unthinkable these days to carry out a non-standard laboratory test without structural sensing. All other engineering activities in this list have yet to include structural sensing so intensively. 
Sensing is used increasingly in construction phases to help reduce risk when structural behavior could exceed a limit state. Since there is little time for data interpretation, measurements of quantities of interest need to be as direct as possible. For example, if web shear strength could be exceeded during the launching of steel bridge girders, the best measurement to make is the load over the launching support since that is directly related to the shear force in the bridge girder. Deflection measurements at the other end of the girder are much less direct, and interpreting such measurements requires estimation of other factors such as temperature and wind effects.

Compared with laboratory research, precision and accuracy requirements for construction-phase sensors are not as critical. Five to ten percent of the measured value is often sufficient. In this activity, challenges include ensuring that sensors remain reliable when subjected to construction-site conditions. Another challenge is related to communication and coordination. Workers have been known to rip out sensor equipment when they have not been informed of its importance. Placement-of-instrumentation information needs to be included in information models that are available to all present on site. Standards for doing this do not exist. Lastly, little work exists on optimal sensor-system design for use during construction and later, in service.

The importance of measuring structures at early stages in their service lives, prior to damage, is being increasingly recognized. Engineers who work in the built environment field often design objects that have orders of magnitude more value than the firms where they work. Furthermore, the most dangerous time in the life of a structure is often during construction. This means that the consequences of design mistakes can be very costly and their impact is felt immediately. Being risk averse, structural engineers justifiably design conservatively using safe behavior models and idealized boundary conditions. Quantification of reserve capacity has the potential to reduce costs due to extension (such as extra bridge lanes), repair, retrofitting, and repurposing (for example, adding a railway line to a road bridge). Perhaps, the most financial gain is realized when replacement can be avoided altogether. Few research studies have concentrated on the general challenge of defining and predicting reserve capacity. Quantifying uncertainties related to behavior models, loading, and environmental effects as well as finding reliable data-interpretation methodologies that include qualitative observations are specific challenges.

Of all the activities mentioned earlier, sensing during some point in service lives is probably the activity that traditionally has involved the most structural sensing. For example, when damage such as rust staining, an earthquake-induced effect, cracking, spalling, and differential settlement, is observed, engineers need to know whether or not structural integrity has been compromised and if so, to what extent. Challenges related to this activity include those mentioned above for structures at early service-life stages as well as modeling the effect of deterioration and further monitoring of its progression.

While damage detection is a prime motivation in other structural sensing fields such as mechanical engineering and aeronautics, it is only of marginal interest to engineers in the built environment (with the exception of those in earthquake engineering). This is because structures and materials used in the built environment are designed to be damage tolerant. Damage most often becomes visible well before a limit state is attained.

Nevertheless, there are situations, such as in zones of medium to high earthquake risk, where measurement for damage detection can be justified. Also, a range of techniques for behaviormodel-free detection of anomalies using signal analysis methods have emerged as inexpensive and fast methods for monitoring of structures for damage detection. Challenges include achieving high enough sensitivity to justify complementarity with visual observation. For example, there is no sense implementing instrumentation for damage detection when it is capable of indicating damage only after damage has been easily visible for some time. Since many sensing strategies are still at this stage, increasing sensitivity in this context is a grand challenge.

Sensing during demolition and dismantlement has similar application and challenges as observed during construction stages. Measurements need to be direct so that data interpretation is fast. An application that is specific to this activity involves sensing that is used to control explosive-induced progressive collapse. In such applications, the moment when sensors cease to provide information needs to be known. There has been very little work in this area and therefore, using sensors for controlled explosions is a grand challenge.

Sensing is an integral part of control systems for active structures. Sensing enables determination of appropriate reactions in challenging environments, such as space, deep sea, polar regions, and in nuclear power facilities. Good sensing leads to structures that can increase their performance with time, structures that can self-diagnose following damage and then adapt to this damage, structures that can mitigate vibrations, and structures that can deploy through multiple degrees of freedom. The grand challenge in this application of structural sensing is matching sensor-system characteristics to the functionalities that are required of the active structure.

Finally, there are several general grand challenges that apply to most of the application areas mentioned above. Without attempting to provide an exhaustive list, some of these are:

- lowering the cost and increasing the reliability of predictions using behavior model-based data interpretation;

- increasing the sensitivity of behavior model-free methods to structural-system changes;

- developing new sensors that can be used in challenging environments;

- using the effects of changes in environmental variables to improve data interpretation;

- quantifying and propagating uncertainties for identification, interpolation, and extrapolation;

- coping with the high levels of systematic uncertainty that are commonly associated with structural behavior models;

- finding interpretation strategies that are robust when uncertainties are not completely known;

- measurement system design including sensor type, position, and multiple design criteria;

- identifying situations of over instrumentation;

- determining when results from other engineering fields are appropriate for application in contexts of structures in the built environment; 
- developing clear scientific arguments intended for practitioners that sensing can be an investment and not a cost;

- including engineering heuristics, contextual information, and qualitative observations in data-interpretation frameworks;

- defining the potential impact of sensing on structural design practice;

- using sensing to develop new types of active structures for new contexts;

- extending active structures to display biomimetic behavior, such as learning, damage detection, automatic adaptation, and damage tolerance.

Meeting these and other challenges cannot be accomplished in a short time. As progress is made, the number of structures that are measured will increase and this will serve as a measure of our success. New sensors will emerge that will help meet many of these challenges and add to the number of useful applications of structural sensing. Ultimately, structural sensing will contribute to a new generation of structures that will inexpensively provide functionalities that contribute to sustainability and quality of life.

Conflict of Interest Statement: The author declares that the research was conducted in the absence of any commercial or financial relationships that could be construed as a potential conflict of interest.

Copyright (c) 2015 Smith. This is an open-access article distributed under the terms of the Creative Commons Attribution License (CC BY). The use, distribution or reproduction in other forums is permitted, provided the original author(s) or licensor are credited and that the original publication in this journal is cited, in accordance with accepted academic practice. No use, distribution or reproduction is permitted which does not comply with these terms. 